\title{
Wave breaking in the short-pulse equation
}

\author{
Yue Liu, Dmitry Pelinovsky, and Anton Sakovich
}

\author{
Communicated by Y. Charles Li, received May 27, 2009.
}

\begin{abstract}
Sufficient conditions for wave breaking are found for the shortpulse equation describing wave packets of few cycles on the ultra-short pulse scale. The analysis relies on the method of characteristics and conserved quantities of the short-pulse equation and holds both on an infinite line and in a periodic domain. Numerical illustrations of the finite-time wave breaking are given in a periodic domain.
\end{abstract}

\section{Contents}

1. Introduction

2. Wave breaking on an infinite line

3. Wave breaking in a periodic domain

4. Numerical evidence of wave breaking

\section{Introduction}

The short-pulse equation,

$$
u_{t x}=u+\frac{1}{6}\left(u^{3}\right)_{x x}, \quad t>0, x \in \mathbb{R},
$$

is a useful and simple approximation of nonlinear wave packets in dispersive media in the limit of few cycles on the ultra-short pulse scale $[\mathbf{1}, \mathbf{1 1}]$. This equation is a dispersive generalization of the following advection equation

$$
u_{t}=\frac{1}{2} u^{2} u_{x}, \quad t>0, x \in \mathbb{R} .
$$

According to the method of characteristics, the advection equation (1.2) exhibits wave breaking in a finite time for any initial data $u(x, 0)=u_{0}(x)$ on an

2000 Mathematics Subject Classification. 35, 76.

Key words and phrases. Wave breaking, ultra-short pulse, short-pulse equation. 
infinite line if $u_{0}(x)$ is continuously differentiable and there is a point $x_{0} \in \mathbb{R}$ such that $u_{0}\left(x_{0}\right) u_{0}^{\prime}\left(x_{0}\right)>0$. This follows from the implicit solution

$$
u(\xi, t)=u_{0}(\xi), \quad x(\xi, t)=\xi-\frac{1}{2} u_{0}^{2}(\xi) t, \quad t>0, \quad \xi \in \mathbb{R},
$$

for any given $u_{0}(x) \in C^{1}(\mathbb{R})$. We say that the finite-time wave breaking occurs if there exists a finite time $T \in(0, \infty)$ such that

$$
\lim _{t \uparrow T} \sup _{x \in \mathbb{R}} u(x, t) u_{x}(x, t)=\infty, \quad \text { while } \quad \lim _{t \uparrow T} \sup _{x \in \mathbb{R}}|u(x, t)|<\infty .
$$

For the simple advection equation (1.2),

$$
T=\inf _{\xi \in \mathbb{R}}\left\{\frac{1}{u_{0}(\xi) u_{0}^{\prime}(\xi)}: \quad u_{0}(\xi) u_{0}^{\prime}(\xi)>0\right\} .
$$

In view of this result, we address the question if the dispersion term $\partial_{x}^{-1} u$ in the short-pulse equation (1.1) can stabilize global dynamics of the advection equation (1.2) at least for small initial data. Local well-posedness of the short-pulse equation on an infinite line was proven in $[\mathbf{1 1}]$.

Theorem 1 (Schäfer \& Wayne, 2004). Let $u_{0} \in H^{2}(\mathbb{R})$. There exists a $T>0$ such that the short-pulse equation (1.1) admits a unique solution

$$
u(t) \in C\left([0, T), H^{2}(\mathbb{R})\right) \cap C^{1}\left([0, T), H^{1}(\mathbb{R})\right)
$$

satisfying $u(0)=u_{0}$. Furthermore, the solution $u(t)$ depends continuously on $u_{0}$.

To extend local solutions into a global solution, Pelinovsky \& Sakovich [9] used the following conserved quantities of the short-pulse equation:

$$
\begin{aligned}
E_{0} & :=\int_{\mathbb{R}} u^{2} d x, \\
E_{1} & :=\int_{\mathbb{R}}\left(\sqrt{1+u_{x}^{2}}-1\right) d x=\int_{\mathbb{R}} \frac{u_{x}^{2}}{1+\sqrt{1+u_{x}^{2}}} d x, \\
E_{2} & :=\int_{\mathbb{R}} \sqrt{1+u_{x}^{2}}\left[\partial_{x}\left(\frac{u_{x}}{\sqrt{1+u_{x}^{2}}}\right)\right]^{2} d x=\int_{\mathbb{R}} \frac{u_{x x}^{2}}{\left(1+u_{x}^{2}\right)^{5 / 2}} d x .
\end{aligned}
$$

If $u(t)$ is a local solution in Theorem 1 , then $E_{0}, E_{1}$, and $E_{2}$ are bounded and constant in time for all $t \in[0, T)$. Global well-posedness of the short-pulse equation on an infinite line was proven in [9] for small initial data in $H^{2}$ satisfying

$$
2 E_{1}+E_{2} \leq\left\|u_{0}^{\prime}\right\|_{L^{2}}^{2}+\left\|u_{0}^{\prime \prime}\right\|_{L^{2}}^{2}<1 .
$$

The condition (1.7) can be sharpen using the scaling transformation for the shortpulse equation (1.1). Let $\alpha \in \mathbb{R}_{+}$be an arbitrary parameter. If $u(x, t)$ is a solution of $(1.1)$, then $\tilde{u}(\tilde{x}, \tilde{t})$ is also a solution of (1.1) with

$$
\tilde{x}=\alpha x, \quad \tilde{t}=\alpha^{-1} t, \quad \tilde{u}(\tilde{x}, \tilde{t})=\alpha u(x, t) .
$$

The conserved quantities transform as follows:

$$
\begin{aligned}
& \tilde{E}_{1}=\int_{\mathbb{R}}\left(\sqrt{1+\tilde{u}_{\tilde{x}}^{2}}-1\right) d \tilde{x}=\alpha \int_{\mathbb{R}}\left(\sqrt{1+u_{x}^{2}}-1\right) d x=\alpha E_{1}, \\
& \tilde{E}_{2}=\int_{\mathbb{R}} \frac{\tilde{u}_{\tilde{x} \tilde{x}}^{2}}{\left(1+\tilde{u}_{\tilde{x}}^{2}\right)^{5 / 2}} d \tilde{x}=\alpha^{-1} \int_{\mathbb{R}} \frac{u_{x x}^{2}}{\left(1+u_{x}^{2}\right)^{5 / 2}} d x=\alpha^{-1} E_{2} .
\end{aligned}
$$


Finding the minimum of $2 \tilde{E}_{1}+\tilde{E}_{2}=2 \alpha E_{1}+\alpha^{-1} E_{2}$ in $\alpha$ gives a sharper sufficient condition on global well-posedness $[\mathbf{9}]$.

Theorem 2 (Pelinovsky \& Sakovich, 2008). Let $u_{0} \in H^{2}(\mathbb{R})$ and $2 \sqrt{2 E_{1} E_{2}}<$ 1. Then the short-pulse equation (1.1) admits a unique global solution $u(t) \in$ $C\left(\mathbb{R}_{+}, H^{2}(\mathbb{R})\right)$ satisfying $u(0)=u_{0}$.

Theorem 2 does not exclude wave breaking in a finite time for large initial data and this paper gives a proof that the wave breaking may occur in the shortpulse equation (1.1). Negating the sufficient condition for global well-posedness in Theorem 2, a necessary condition for the wave breaking follows: the wave breaking may occur in the short-pulse equation (1.1) with the initial data $u_{0} \in H^{2}$ only if $2 \sqrt{2 E_{1} E_{2}} \geq 1$. We shall find a sufficient condition for the wave breaking in the short-pulse equation (1.1).

Unlike the previous work in [9], we will not be using conserved quantity $E_{2}$ but will rely on the conservation of $E_{0}, E_{1}$, and the energy

$$
E_{-1}:=\int_{\mathbb{R}}\left[\left(\partial_{x}^{-1} u\right)^{2}-\frac{1}{12} u^{4}\right] d x .
$$

Here $\partial_{x}^{-1} u$ is defined from a local solution $u$ by

$$
\partial_{x}^{-1} u:=\int_{-\infty}^{x} u\left(x^{\prime}, t\right) d x^{\prime}=-\int_{x}^{\infty} u\left(x^{\prime}, t\right) d x^{\prime}=\frac{1}{2}\left(\int_{-\infty}^{x}-\int_{x}^{\infty}\right) u\left(x^{\prime}, t\right) d x^{\prime},
$$

thanks to the zero-mass constraint $\int_{\mathbb{R}} u(x, t) d x=0$ for all $t \in(0, T)$. (Note that the initial data $u_{0}$ does not have generally to satisfy the zero-mass constraint $\int_{\mathbb{R}} u_{0}(x) d x=0$.)

Thanks to the Sobolev inequality, $\|u\|_{L^{4}} \leq C\|u\|_{H^{1}}$ for some $C>0$, the quantity $E_{-1}$ is bounded if $u \in H^{2}(\mathbb{R}) \cap \dot{H}^{-1}(\mathbb{R})$, where $\dot{H}^{-1}$ is defined by its norm

$$
\|u\|_{\dot{H}^{-1}}:=\left\|\partial_{x}^{-1} u\right\|_{L^{2}} .
$$

(Note that if $u \in H^{2}(\mathbb{R}) \cap \dot{H}^{-1}(\mathbb{R})$, then $\int_{\mathbb{R}} u(x) d x=0$ is satisfied.) Our main result on the wave breaking on an infinite line is formulated as follows.

Theorem 3. Let $u_{0} \in H^{2}(\mathbb{R}) \cap \dot{H}^{-1}(\mathbb{R})$ and $T$ be the maximal existence time of Theorem 1. Let

$$
\begin{aligned}
& F_{1}:=\frac{1}{\sqrt{2}}\left(E_{1}^{2}+\left(8 E_{0} E_{1}+E_{1}^{4}\right)^{1 / 2}\right)^{1 / 2}, \\
& F_{0}:=\frac{1}{\sqrt{2}}\left(E_{0}+E_{-1}+\frac{1}{12} E_{0} F_{1}^{2}\right)^{1 / 2},
\end{aligned}
$$

and assume that there exists $x_{0} \in \mathbb{R}$ such that $u_{0}\left(x_{0}\right) u_{0}^{\prime}\left(x_{0}\right)>0$ and

$$
\begin{array}{rlrl}
\text { either } & & \left|u_{0}^{\prime}\left(x_{0}\right)\right| & >\left(\frac{F_{1}^{2}}{4 F_{0}}\right)^{1 / 3}, \\
& \left|u_{0}\left(x_{0}\right)\right|\left|u_{0}^{\prime}\left(x_{0}\right)\right|^{2}>F_{1}+\left(2 F_{0}\left|u_{0}^{\prime}\left(x_{0}\right)\right|^{3}-\frac{1}{2} F_{1}^{2}\right)^{1 / 2}, \\
\text { or } & \left|u_{0}^{\prime}\left(x_{0}\right)\right| \leq\left(\frac{F_{1}^{2}}{4 F_{0}}\right)^{1 / 3}, \quad\left|u_{0}\left(x_{0}\right)\right|\left|u_{0}^{\prime}\left(x_{0}\right)\right|^{2}>F_{1} .
\end{array}
$$


Then $T<\infty$, so that the solution $u(t) \in C\left([0, T), H^{2}(\mathbb{R}) \cap \dot{H}^{-1}(\mathbb{R})\right)$ of the shortpulse equation (1.1) blows up in the sense of

$$
\lim _{t \uparrow T} \sup _{x \in \mathbb{R}} u(x, t) u_{x}(x, t)=\infty, \quad \text { while } \quad \lim _{t \uparrow T}\|u(\cdot, t)\|_{L^{\infty}} \leq F_{1} .
$$

REMARK 1. The quantities $F_{0}$ and $F_{1}$ in Theorem 3 can be defined by

$$
F_{1}:=\sup _{t \in[0, T)}\|u(\cdot, t)\|_{L^{\infty}}, \quad F_{0}:=\sup _{t \in[0, T)}\left\|\partial_{x}^{-1} u(\cdot, t)\right\|_{L^{\infty}}
$$

Note that the scaling transformation (1.8) gives

$$
\|\tilde{u}(\cdot, \tilde{t})\|_{L^{\infty}}=\alpha\|u(\cdot, t)\|_{L^{\infty}}, \quad\left\|\partial_{\tilde{x}}^{-1} \tilde{u}(\cdot, \tilde{t})\right\|_{L^{\infty}}=\alpha^{2}\left\|\partial_{x}^{-1} u(\cdot, t)\right\|_{L^{\infty}},
$$

so that the sufficient condition of Theorem 3 with new definitions of $F_{1}$ and $F_{0}$ is invariant in $\alpha$. We note, however, that, while the bound on $F_{1}$ in Theorem 3 scales correctly as $\tilde{F}_{1}=\alpha F_{1}$, the bound on $F_{0}$ is not correctly scaled in $\alpha$ because

$$
\tilde{E}_{0}=\alpha^{3} E_{0}, \quad \tilde{E}_{-1}=\alpha^{5} E_{-1} .
$$

This is an artefact of using Sobolev embedding in Lemma 3 below.

To prove Theorem 3, we shall adopt the method of characteristics and proceed with apriori differential estimates. Our results remain valid in a periodic domain, where Theorem 3 is replaced by Theorem 4 below. The technique of characteristics and apriori differential estimates has been applied for wave breaking in other nonlinear wave equations, see $[\mathbf{2}, \mathbf{3}, \mathbf{4}, \mathbf{5}, \mathbf{6}, \mathbf{7}, \mathbf{1 2}]$ for an incomplete list of references.

We emphasize that, unlike the previous work in [9], we avoid using a transformation between the short-pulse equation (1.1) and the integrable sine-Gordon equation in characteristic coordinates. Our proof of the wave breaking for the shortpulse equation (1.1) does not suggest that there exists a similar wave breaking for the sine-Gordon equation, it is rather the breaking of the coordinate transformation between the two equations. On a similar note, we do not use the integrability properties of the short-pulse equation (1.1) such as the Lax pair, the inverse scattering transform method, the bi-Hamiltonian formulation, and the existence of exact soliton solutions.

The article is constructed as follows. The proof of Theorem 3 is given in Section 2. Section 3 reports extension of Theorem 3 to a periodic domain. Section 4 contains numerical evidences of the finite-time wave breaking in a periodic domain.

Acknowledgement. The work of Y. Liu is partially supported by the NSF grant DMS-0906099. The work of D. Pelinovsky is supported by the NSERC grant RGPIN238931-06. The work of A. Sakovich is supported by the McMaster graduate scholarship.

\section{Wave breaking on an infinite line}

Let us rewrite the Cauchy problem for the short-pulse equation on an infinite line in the form

$$
\left\{\begin{array}{l}
u_{t}=\frac{1}{2} u^{2} u_{x}+\partial_{x}^{-1} u, \quad x \in \mathbb{R}, \quad t>0 \\
u(x, 0)=u_{0}(x), \quad x \in \mathbb{R},
\end{array}\right.
$$

where $\partial_{x}^{-1} u:=\int_{-\infty}^{x} u\left(x^{\prime}, t\right) d x^{\prime}$. In what follows, we use both notations $u(t)$ and $u(x, t)$ for the same solution of the Cauchy problem (2.1). Local existence of solutions with the conservation of $E_{-1}$ and $E_{0}$ is described by the following result. 
LEMMA 1. Let $u_{0} \in H^{s}(\mathbb{R}) \cap \dot{H}^{-1}(\mathbb{R}), s \geq 2$. There exist a maximal time $T=T\left(u_{0}\right)>0$ and a unique solution $u(x, t)$ to the Cauchy problem (2.1) such that

$$
u(t) \in C\left([0, T), H^{s}(\mathbb{R}) \cap \dot{H}^{-1}(\mathbb{R})\right) \cap C^{1}\left([0, T), H^{s-1}(\mathbb{R})\right)
$$

satisfying $u(0)=u_{0}$. Moreover, the solution $u(t)$ depends continuously on the initial data $u_{0}$ and the values of $E_{-1}, E_{0}$, and $E_{1}$ in (1.4), (1.5), and (1.9) are constant on $[0, T)$.

Proof. If $u_{0} \in H^{s}(\mathbb{R}) \cap \dot{H}^{-1}(\mathbb{R}), s \geq 2$, then $\partial_{x}^{-1} u_{0} \in H^{s+1}(\mathbb{R})$, so that $\int_{\mathbb{R}} u_{0}(x) d x=0$. By the theorem of Schäfer \& Wayne $[\mathbf{1 1}]$, there exists a solution

$$
u(t) \in C\left([0, T), H^{s}(\mathbb{R})\right) \cap C^{1}\left([0, T), H^{s-1}(\mathbb{R})\right)
$$

of the short-pulse equation (1.1), so that

$$
\partial_{x}^{-1} u(t):=u_{t}-\frac{1}{2} u^{2} u_{x} \in C\left((0, T), H^{s-1}(\mathbb{R})\right)
$$

Therefore, $u(t) \in C\left([0, T), H^{s}(\mathbb{R}) \cap \dot{H}^{-1}(\mathbb{R})\right)$ in view of boundness of $\left\|u_{0}\right\|_{\dot{H}^{-1}}$. Because $f \in H^{s}(\mathbb{R}), s \geq 1$ implies $\lim _{|x| \rightarrow \infty} f(x)=0$, the zero-mass constraint holds in the form

$$
\int_{\mathbb{R}} u(x, t) d x=0, \quad t \in[0, T) .
$$

Let us define

$$
\partial_{x}^{-2} u(t):=\left(\partial_{x}^{-1} u\right)_{t}-\frac{1}{6} u^{3}
$$

By the zero-mass constraint (2.2) and uniqueness of the solution $u(t)$ for any $t \in$ $[0, T)$, we obtain

$$
\lim _{|x| \rightarrow \infty} \partial_{x}^{-2} u(x, t)=0, \quad t \in[0, T)
$$

Using balance equations for the densities of $E_{-1}, E_{0}$, and $E_{1}$, we write

$$
\begin{aligned}
{\left[\left(\partial_{x}^{-1} u\right)^{2}-\frac{1}{12} u^{4}\right]_{t} } & =\left[\left(\partial_{x}^{-2} u\right)^{2}-\frac{1}{36} u^{6}\right]_{x}, \\
\left(u^{2}\right)_{t} & =\left(\left(\partial_{x}^{-1} u\right)^{2}+\frac{1}{4} u^{4}\right)_{x}, \\
\left(\sqrt{1+u_{x}^{2}}-1\right)_{t} & =\frac{1}{2}\left(u^{2} \sqrt{1+u_{x}^{2}}\right)_{x} .
\end{aligned}
$$

Integrating the balance equation in $x \in \mathbb{R}$ for any $t \in[0, T)$, we complete the proof that $E_{-1}, E_{0}$, and $E_{1}$ are bounded and constant on $[0, T)$.

REMARK 2. The maximal existence time $T>0$ in Lemma 1 is independent of $s \geq 2$ in the following sense. If $u_{0} \in H^{s}(\mathbb{R}) \cap H^{s^{\prime}}(\mathbb{R}) \cap \dot{H}^{-1}(\mathbb{R})$ for $s, s^{\prime} \geq 2$ and $s \neq s^{\prime}$, then

$$
u(t) \in C\left([0, T), H^{s}(\mathbb{R}) \cap \dot{H}^{-1}(\mathbb{R})\right) \cap C^{1}\left([0, T), H^{s-1}(\mathbb{R})\right)
$$

and

$$
u(t) \in C\left(\left[0, T^{\prime}\right), H^{s^{\prime}}(\mathbb{R}) \cap \dot{H}^{-1}(\mathbb{R})\right) \cap C^{1}\left(\left[0, T^{\prime}\right), H^{s^{\prime}-1}(\mathbb{R})\right)
$$

with the same $T^{\prime}=T$. See Yin [12] for standard arguments.

By using the local well-posedness result in Lemma 1 and energy estimates, we obtain a precise blow-up scenario of the solutions to the Cauchy problem (2.1). 
LEMMA 2. Let $u_{0} \in H^{2}(\mathbb{R}) \cap \dot{H}^{-1}(\mathbb{R})$ and $u(t)$ be a solution of the Cauchy problem (2.1) in Lemma 1. The solution blows up in a finite time $T \in(0, \infty)$ in the sense of $\lim _{t \uparrow T}\|u(\cdot, t)\|_{H^{2}}=\infty$ if and only if

$$
\lim _{t \uparrow T} \sup _{x \in \mathbb{R}} u(x, t) u_{x}(x, t)=+\infty .
$$

ProOF. We only need to prove the necessary condition, since the singularity in $u(x, t) u_{x}(x, t)$ as $t \uparrow T$ implies the singularity in $\|u(\cdot, t)\|_{H^{2}}$ as $t \uparrow T$. Assume a finite maximal existence time $T \in(0, \infty)$ and suppose, by the contradiction, that there is $M>0$ such that

$$
\sup _{x \in \mathbb{R}} u(x, t) u_{x}(x, t) \leq M<\infty, \quad \forall t \in[0, T) .
$$

Applying density arguments, we approximate the initial value $u_{0} \in H^{2}(\mathbb{R})$ by functions $u_{0}^{n} \in H^{3}(\mathbb{R}), n \geq 1$, so that $\lim _{n \rightarrow \infty} u_{0}^{n}=u_{0}$. Furthermore, write $u^{n}(t)$ for the solution of the Cauchy problem (2.1) with initial data $u_{0}^{n}$. Using the regularity result proved in Lemma 1 for $s=3$, it follows from Sobolev's embedding that, if $u^{n}(t) \in C\left([0, T), H^{3}(\mathbb{R}) \cap \dot{H}^{-1}(\mathbb{R})\right)$, then $u^{n}(x, t)$ is a twice continuously differentiable function of $x$ on $\mathbb{R}$ for any $t \in[0, T)$. It is then deduced from the short-pulse equation (1.1) that

$$
\frac{d}{d t} \int_{\mathbb{R}}\left(u_{x}^{n}\right)^{2} d x=\int_{\mathbb{R}} u^{n}\left(u_{x}^{n}\right)^{3} d x \leq M \int_{\mathbb{R}}\left(u_{x}^{n}\right)^{2} d x
$$

and

$$
\frac{d}{d t} \int_{\mathbb{R}}\left(u_{x x}^{n}\right)^{2} d x=5 \int_{\mathbb{R}} u^{n} u_{x}^{n}\left(u_{x x}^{n}\right)^{2} d x \leq 5 M \int_{\mathbb{R}}\left(u_{x x}^{n}\right)^{2} d x .
$$

The Gronwall inequality yields for all $t \in[0, T)$,

$$
\left\|u_{x}^{n}(\cdot, t)\right\|_{L^{2}} \leq\left\|\left(u_{0}^{n}\right)^{\prime}\right\|_{L^{2}} e^{\frac{M}{2} t}, \quad\left\|u_{x x}^{n}(\cdot, t)\right\|_{L^{2}} \leq\left\|\left(u_{0}^{n}\right)^{\prime \prime}\right\|_{L^{2}} e^{\frac{5}{2} M t} .
$$

Since $\left\|u_{0}^{n}\right\|_{H^{2}}$ converges to $\left\|u_{0}\right\|_{H^{2}}$ as $n \rightarrow \infty$, we infer from the continuous dependence of the local solution $u(t)$ on initial data $u_{0}$ that $\|u(\cdot, t)\|_{H^{2}}$ remains bounded on $[0, T)$ for the solution $u(t)$ in Lemma 1 . Therefore, the contradiction is obtained and either $T$ is not a maximal existence time or the bound (2.3) is not valid on $[0, T)$.

We also show that the blow-up of Lemma 2 is the wave breaking in the sense of condition (1.3). In other words, both $\|u(\cdot, t)\|_{L^{\infty}}$ and $\left\|\partial_{x}^{-1} u(\cdot, t)\right\|_{L^{\infty}}$ are uniformly bounded for all $t \in[0, T)$.

LEMma 3. Let $u_{0} \in H^{2}(\mathbb{R}) \cap \dot{H}^{-1}(\mathbb{R})$ and $T>0$ be the maximal existence time of the solution $u(x, t)$ in Lemma 1. Then,

$$
\|u(\cdot, t)\|_{L^{\infty}} \leq F_{1}, \quad\left\|\partial_{x}^{-1} u(\cdot, t)\right\|_{L^{\infty}} \leq F_{0}, \quad t \in[0, T),
$$

where

$$
\begin{aligned}
& F_{1}:=\frac{1}{\sqrt{2}}\left(E_{1}^{2}+\left(8 E_{0} E_{1}+E_{1}^{4}\right)^{1 / 2}\right)^{1 / 2} \\
& F_{0}:=\frac{1}{\sqrt{2}}\left(E_{0}+E_{-1}+\frac{1}{12} E_{0} F_{1}^{2}\right)^{1 / 2} .
\end{aligned}
$$


Proof. For all $t \in[0, T)$ and the solution $u(x, t)$, we have

$$
\begin{aligned}
u^{2}(x, t) & =\left|\int_{-\infty}^{x} u u_{x} d x-\int_{x}^{\infty} u u_{x} d x\right| \\
& \leq \int_{\mathbb{R}} \frac{|u|\left|u_{x}\right|}{\sqrt{1+\sqrt{1+u_{x}^{2}}}} \sqrt{1+\sqrt{1+u_{x}^{2}}} d x \\
& \leq E_{1}^{1 / 2}\left(\int_{\mathbb{R}} u^{2}\left(2+\sqrt{1+u_{x}^{2}}-1\right) d x\right)^{1 / 2} \\
& \leq E_{1}^{1 / 2}\left(2 E_{0}+E_{1}\|u(\cdot, t)\|_{L^{\infty}}^{2}\right)^{1 / 2} .
\end{aligned}
$$

As a result, we obtain

$$
\|u(\cdot, t)\|_{L^{\infty}}^{4} \leq 2 E_{0} E_{1}+E_{1}^{2}\|u(\cdot, t)\|_{L^{\infty}}^{2},
$$

so that bound (2.5) is found from the quadratic equation on $\|u(\cdot, t)\|_{L^{\infty}}^{2}$. On the other hand, we have

$$
\begin{aligned}
\left\|\partial_{x}^{-1} u(\cdot, t)\right\|_{H^{1}}^{2} & =\|u(\cdot, t)\|_{L^{2}}^{2}+\left\|\partial_{x}^{-1} u(\cdot, t)\right\|_{L^{2}}^{2} \\
& =E_{0}+E_{-1}+\frac{1}{12}\|u(\cdot, t)\|_{L^{4}}^{4} \\
& \leq E_{0}+E_{-1}+\frac{1}{12} E_{0}\|u(\cdot, t)\|_{L^{\infty}}^{2} .
\end{aligned}
$$

Using the Sobolev inequality $\left\|\partial_{x}^{-1} u\right\|_{L^{\infty}} \leq \frac{1}{\sqrt{2}}\left\|\partial_{x}^{-1} u\right\|_{H^{1}}$ and bound (2.5), we obtain bound (2.6).

Let us introduce a continuous family of characteristics for solutions of the Cauchy problem (2.1). Let $\xi \in \mathbb{R}, t \in[0, T)$, where $T$ is the maximal existence time in Lemma 1 , and denote

$$
x=X(\xi, t), \quad u(x, t)=U(\xi, t), \quad \partial_{x}^{-1} u(x, t)=G(\xi, t)
$$

so that

$$
\left\{\begin{array} { l } 
{ \dot { X } ( t ) = - \frac { 1 } { 2 } U ^ { 2 } , } \\
{ X ( 0 ) = \xi }
\end{array} \quad \left\{\begin{array}{l}
\dot{U}(t)=G \\
U(0)=u_{0}(\xi)
\end{array}\right.\right.
$$

where dots denote derivatives with respect to time $t$ on a particular characteristics $x=X(\xi, t)$ for a fixed $\xi \in \mathbb{R}$. Applying classical results in the theory of ordinary differential equations, we obtain the following useful result about the solutions of the initial-value problem (2.8). Conserved quantities $E_{-1}$ and $E_{0}$ of the Cauchy problem (2.1) are used to control values of $U$ and $G$ on the family of characteristics.

LEMMA 4. Let $u_{0} \in H^{2}(\mathbb{R}) \cap \dot{H}^{-1}(\mathbb{R})$ and $T>0$ be the maximal existence time of the solution $u(t)$ in Lemma 1. Then there exists a unique solution $X(\xi, t) \in$ $C^{1}(\mathbb{R} \times[0, T))$ to the initial-value problem (2.8). Moreover, the map $X(\cdot, t): \mathbb{R} \mapsto \mathbb{R}$ is an increasing diffeomorphism for any $t \in[0, T)$ with

$$
\partial_{\xi} X(\xi, t)=\exp \left(-\int_{0}^{t} u(X(\xi, s), s) u_{x}(X(\xi, s), s) d s\right)>0, \quad t \in[0, T), \quad \xi \in \mathbb{R} .
$$


Proof. Existence and uniqueness of $X(\xi, t) \in C^{1}(\mathbb{R},[0, T))$ follows from the integral equation

$$
X(\xi, t)=\xi-\frac{1}{2} \int_{0}^{t} U^{2}(\xi, s) d s, \quad t \in[0, T), \quad \xi \in \mathbb{R}
$$

since $U(\xi, t) \in C(\mathbb{R},[0, T))$ for the solution $u(t)$ in Lemma 1. Using the chain rule, we obtain

$$
\partial_{\xi} \dot{X}(\xi, t)=-W(\xi, t) \partial_{\xi} X(\xi, t) \quad \Rightarrow \quad \partial_{\xi} X(\xi, t)=\exp \left(-\int_{0}^{t} W(\xi, s) d s\right),
$$

where $W(\xi, t)=u(X(\xi, t), t) u_{x}(X(\xi, t), t) \in C(\mathbb{R},[0, T))$. Therefore, $\partial_{\xi} X(\xi, t)>0$ for all $t \in[0, T)$ and $\xi \in \mathbb{R}$.

Let

$$
V(\xi, t)=u_{x}(X(\xi, t), t), \quad W(\xi, t)=u(X(\xi, t), t) u_{x}(X(\xi, t), t) \equiv U(\xi, t) V(\xi, t)
$$

and compute their rate of changes along the family of characteristics

$$
\left\{\begin{array}{l}
\dot{V}=V W+U \\
\dot{W}=W^{2}+V G+U^{2}
\end{array}\right.
$$

Let $F_{0}, F_{1}>0$ be fixed in terms of conserved quantities $E_{-1}, E_{0}$, and $E_{1}$ as in Lemma 3 and assume that there exists $\xi_{0} \in \mathbb{R}$ such that $W\left(\xi_{0}, 0\right)>0$ and

$$
\begin{aligned}
\text { either } & & \left|V\left(\xi_{0}, 0\right)\right|>\left(\frac{F_{1}^{2}}{4 F_{0}}\right)^{1 / 3}, \\
& & \left|V\left(\xi_{0}, 0\right)\right| W\left(\xi_{0}, 0\right)>F_{1}+\left(2 F_{0}\left|V\left(\xi_{0}, 0\right)\right|^{3}-\frac{1}{2} F_{1}^{2}\right)^{1 / 2}, \\
\text { or } & & \left|V\left(\xi_{0}, 0\right)\right| \leq\left(\frac{F_{1}^{2}}{4 F_{0}}\right)^{1 / 3}, \quad\left|V\left(\xi_{0}, 0\right)\right| W\left(\xi_{0}, 0\right)>F_{1} .
\end{aligned}
$$

Because of the invariance of system (2.9) with respect to

$$
G \rightarrow-G, \quad U \rightarrow-U, \quad V \rightarrow-V, \quad W \rightarrow W
$$

it is sufficient to consider the case with $V\left(\xi_{0}, 0\right)>0$. We will prove that, under the conditions above, $V\left(\xi_{0}, t\right)$ and $W\left(\xi_{0}, t\right)$ remain positive and monotonically increasing functions for all $t>0$, for which they are bounded, so that $V\left(\xi_{0}, t\right)$ and $W\left(\xi_{0}, t\right)$ satisfy the apriori differential estimates

$$
\left\{\begin{array}{l}
\dot{V} \geq V W-F_{1} \\
\dot{W} \geq W^{2}-V F_{0}
\end{array}\right.
$$

In what follows, we use $V(t)$ and $W(t)$ instead of $V\left(\xi_{0}, t\right)$ and $W\left(\xi_{0}, t\right)$ for a particular $\xi_{0} \in \mathbb{R}$. The following lemma establishes sufficient conditions on the initial point $(V(0), W(0))$ that ensure that a lower solution satisfying

$$
\left\{\begin{array}{l}
\dot{V}=V W-F_{1} \\
\dot{W}=W^{2}-V F_{0}
\end{array}\right.
$$

goes to infinity in a finite time. 
Lemma 5. Assume that the initial data for system (2.11) satisfy

$$
\begin{aligned}
& \text { either } \quad V(0)>\left(\frac{F_{1}^{2}}{4 F_{0}}\right)^{1 / 3} \\
& V(0) W(0)>F_{1}+\left(2 F_{0} V^{3}(0)-\frac{1}{2} F_{1}^{2}\right)^{1 / 2}, \\
& \text { or } \quad 0<V(0) \leq\left(\frac{F_{1}^{2}}{4 F_{0}}\right)^{1 / 3}, \quad V(0) W(0)>F_{1} \text {. }
\end{aligned}
$$

Then the trajectory of system (2.11) blows up in a finite time $t_{*} \in(0, \infty)$ such that $V(t)$ and $W(t)$ are positive and monotonically increasing for all $t \in\left[0, t_{*}\right)$ and there is $C>0$ such that

$$
\lim _{t \uparrow t_{*}} V(t)=\infty, \quad \lim _{t \uparrow t_{*}} W(t)=\infty, \quad \text { and } \quad \lim _{t \uparrow t_{*}}\left(t_{*}-t\right) V(t)=C .
$$

Moreover, $t_{*}$ is bounded by

$$
t_{*} \leq \frac{V(0)}{\min \left\{\dot{V}(0),\left(\dot{V}^{2}(0)-2 F_{0} V^{3}(0)+\frac{1}{2} F_{1}^{2}\right)^{1 / 2}\right\}}
$$

PROOF. Let us first consider the homogeneous version of system (2.11) for $F_{1}=0$, that is

$$
\left\{\begin{array}{l}
\dot{V}=V W \\
\dot{W}=W^{2}-V F_{0} .
\end{array}\right.
$$

Of course, $F_{1}$ is never zero, otherwise $E_{1}=0$. This case is used merely for illustration, since explicit solutions can be obtained for $F_{1}=0$, whereas qualitative analysis has to be developed for $F_{1} \neq 0$. System $(2.16)$ is integrable since

$$
W=\frac{\dot{V}}{V} \Rightarrow \frac{d}{d t}\left(\frac{\dot{V}}{V^{2}}\right)=-F_{0} \Rightarrow \dot{V}=V^{2}\left(C-F_{0} t\right),
$$

where $C=W(0) / V(0)$. Integrating the last equation for $V(t)$, we obtain the explicit solution of the truncated system,

$$
V(t)=\frac{V(0)}{1-C V(0) t+\frac{1}{2} F_{0} V(0) t^{2}}, \quad W(t)=\left(C-F_{0} t\right) V(t) .
$$

The solution reaches infinity in a finite time $t_{*} \in(0, \infty)$ if $V(0)>0$ and $C^{2} V(0)>$ $2 F_{0}$. Note that these conditions coincide with condition $(2.12)$ for $F_{1}=0$. Also note that $t_{*}$ is the first positive root of $1-C V(0) t+\frac{1}{2} F_{0} V(0) t^{2}=0$ so that $U\left(t_{*}\right)=C-F_{0} t_{*}>0$ and

$$
\begin{aligned}
& t_{*}=\frac{W(0)-\sqrt{W^{2}(0)-2 F_{0} V(0)}}{F_{0} V(0)}= \\
& \frac{2 V(0)}{\dot{V}(0)+\sqrt{\dot{V}^{2}(0)-2 F_{0} V^{3}(0)}} \leq \frac{V(0)}{\sqrt{\dot{V}^{2}(0)-2 F_{0} V^{3}(0)}} .
\end{aligned}
$$

Consider now the full system (2.11). Let

$$
V=\frac{1}{x}, \quad W=\frac{y}{x}
$$


and rewrite the system in the form

$$
\left\{\begin{array}{l}
\dot{x}=F_{1} x^{2}-y \\
\dot{y}=F_{1} x y-F_{0} .
\end{array}\right.
$$

Expressing $y$ from the system, we can rewrite it in the scalar form

$$
y=F_{1} x^{2}-\dot{x} \Rightarrow \ddot{x}=f(x)+3 F_{1} x \dot{x},
$$

where $f(x)=F_{0}-F_{1}^{2} x^{3}$. The only critical point of equation $(2.18)$ is $(x, \dot{x})=\left(x_{0}, 0\right)$ where $x_{0}=\left(F_{0} / F_{1}^{2}\right)^{1 / 3}$ is the root of $f(x)$.

We need to show that there is a domain $D \subset \mathbb{R}^{2}$ in the phase plane $(x, \dot{x})$, so that all initial data in $D$ generate trajectories in $D$ that cross the vertical line $x=0$ in a finite time. To do so, we construct a Lyapunov function for equation (2.18) in the form

$$
E(x, \dot{x})=\frac{1}{2} \dot{x}^{2}-F_{0} x+\frac{1}{4} F_{1}^{2} x^{4} .
$$

The function $E(x, \dot{x})$ has a global minimum at $\left(x_{0}, 0\right)$. For any solution $x(t)$, we have

$$
\frac{d}{d t} E(x, \dot{x})=3 F_{1} x \dot{x}^{2}>0 \text { for } \quad x>0 .
$$

The zero level of the Lyapunov function $E(x, \dot{x})=0$ passes through the points $(0,0)$ and $\left(x_{*}, 0\right)$, where $x_{*}=4^{1 / 3} x_{0}>x_{0}$ (see Figure 1$)$. It is clear that $E(x, \dot{x})>0$ in the domain

$$
D=\{(x, \dot{x}): \quad x>0, \quad \dot{x}<\sigma(x)\}
$$

where

$$
\sigma(x)=\left\{\begin{array}{cl}
-\left(2 F_{0} x-\frac{1}{2} F_{1}^{2} x^{4}\right)^{1 / 2}, & x \in\left(0, x_{*}\right), \\
0, & x \in\left[x_{*}, \infty\right) .
\end{array}\right.
$$

We note that the condition $(x, \dot{x}) \in D$ is equivalent to the condition

$$
\begin{cases}0<x<x_{*}, & y>F_{1} x^{2}+\left(2 F_{0} x-\frac{1}{2} F_{1}^{2} x^{4}\right)^{1 / 2}, \\ x>x_{*}, & y>F_{1} x^{2},\end{cases}
$$

which is nothing but the set of conditions (2.12) and (2.13) at $t=0$. By continuity, if $(x, \dot{x}) \in D$ at $t=0$, then $(x, \dot{x})$ remains in $D$ for some time $t>0$.

No critical points of system (2.18) are located in $D$ and $x(t)$ is decreasing function for any $t>0$ as long as the trajectory stays in $D$. Recall that $E(x, \dot{x})>0$ and $\frac{d}{d t} E(x, \dot{x})>0$ for any $(x, \dot{x}) \in D$. A trajectory in $D$ can not cross $\dot{x}=\sigma(x)$ because $E(x, \sigma(x))=0$ for $x \in\left(0, x_{*}\right)$ and $\ddot{x}=f(x)+3 F_{1} x \dot{x}<0$ for $x>x_{*}$ and $\dot{x}<0$. Therefore, the trajectory either reaches $x=0$ in a finite time $t_{*} \in(0, \infty)$ or escapes to $\dot{x}=-\infty$ for $x>0$. To eliminate the last possibility, we note that

$$
\frac{d}{d t}\left(\dot{x}-\frac{3 F_{1}}{2} x^{2}\right)=f(x)
$$

so that

$$
\dot{x}(t) \geq \dot{x}(0)-\frac{3 F_{1}}{2} x^{2}(0)+t f(x(0))>-\infty
$$

for any finite time interval. Moreover, $\dot{x}$ is bounded from zero in $D$ by the level curve $E(x, \dot{x})=E(x(0), \dot{x}(0))$, which is a convex curve in $D$. Therefore,

$$
\dot{x}(t) \leq \max \{\dot{x}(0), \rho\}, \quad t>0, \quad \text { as long as } \quad x>0,
$$




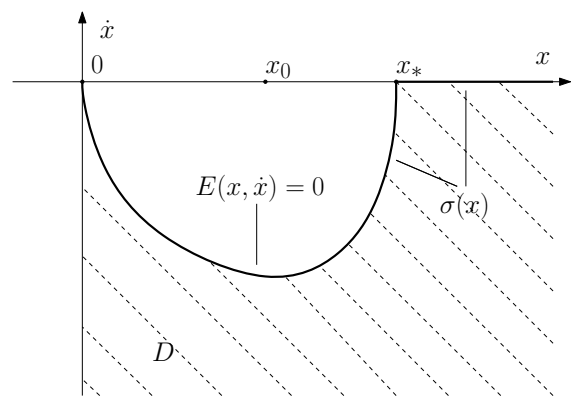

Figure 1. Domain $D$ in the phase plane $(x, \dot{x})$ of equation $(2.18)$.

where $\rho<0$ is uniquely found from $E(0, \rho)=E(x(0), \dot{x}(0))$, that is from the point of intersection of the level curve of $E(x, \dot{x})=E(x(0), \dot{x}(0))$ with the negative $\dot{x}$-axis. Therefore,

$$
x(t) \leq x(0)+t \max \{\dot{x}(0), \rho\},
$$

so that $x(t)$ reaches $x=0$ in a finite time $t_{*} \in(0, \infty)$ for any trajectory in $D$. Moreover, finding $\rho$ explicitly gives the bound on the blow-up time

$$
\begin{cases}0<x<x_{*}, & t_{*} \leq \frac{x(0)}{\left(\dot{x}^{2}(0)-2 F_{0} x(0)+\frac{1}{2} F_{1}^{2} x^{4}(0)\right)^{1 / 2}}, \\ x>x_{*}, & t_{*} \leq \frac{x(0)}{\dot{x}(0) \mid}\end{cases}
$$

which becomes bound (2.15) after the return back to variable $V(t)$.

Since $V=x^{-1}, W=y x^{-1}$, and $y=F_{1} x^{2}-\dot{x}>0$, we have $\lim _{t \uparrow t_{*}} V(t)=\infty$ and $\lim _{t \uparrow t_{*}} W(t)=\infty$ for any trajectories in $D$. Since $\dot{x}<0$ for the trajectory in $D$, we also have $x(t) \sim\left(t_{*}-t\right)$ as $t \rightarrow t_{*}$ so that there exists $C>0$ such that $\lim _{t \uparrow t_{*}}\left(t_{*}-t\right) V(t)=C$. It remains to show that $V(t)$ and $W(t)$ are monotonically increasing functions on $\left[0, t_{*}\right)$. To do so, we write

$$
\begin{aligned}
\dot{V} & =V W-F_{1}=-\frac{\dot{x}}{x^{2}}, \\
\dot{W} & =W^{2}-F_{0} V=\frac{g(x, \dot{x})}{x^{2}},
\end{aligned}
$$

where

$$
g(x, \dot{x})=\dot{x}^{2}-2 F_{1} x^{2} \dot{x}-x f(x) .
$$

For any trajectory in $D, \dot{x}(t)<0$ so that $\dot{V}(t)>0$. Furthermore, since $g(x, \dot{x})$ is zero at a curve outside the domain $D$, because

$$
g(x, \sigma(x))=F_{0} x+\frac{1}{2} F_{1}^{2} x^{4}+2 F_{1} x^{2}|\sigma(x)|>0, \quad x \in\left(0, x_{*}\right],
$$

then $\dot{W}(t)>0$ for any trajectory in $D$.

Recalling that $W=U V$ in system (2.9) and using the bound (2.4), we obtain the upper bound for any solution at the family of characteristics

$$
\dot{V}=U V^{2}+U \leq F_{1} V^{2}+F_{1} \text {. }
$$

We can now show that any upper solution with $V(0)>0$ goes to infinity in a finite time. 
Lemma 6. Consider

$$
\dot{V}(t)=F_{1} V^{2}+F_{1}
$$

with $V(0)>0$. There exists $t_{*} \in(0, \infty)$ such that $V(t)$ is positive, monotonically increasing for all $t \in\left[0, t_{*}\right)$ and there exists $C>0$ such that

$$
\lim _{t \uparrow t_{*}}\left(t_{*}-t\right) V(t)=C \text {. }
$$

Moreover, $t_{*} \leq 1 / W(0)$.

Proof. Since $\dot{V}>0$ for any $V \in \mathbb{R}, V(t)$ is monotonically increasing function. To show that $V(t)$ reaches $\infty$ in a finite time, one can integrate the separable equation (2.20) explicitly and obtain

$$
V(t)=\tan \left(\arctan V(0)+F_{1} t\right)
$$

so that

$$
t_{*}=\frac{\pi / 2-\arctan V(0)}{F_{1}} \leq \frac{1}{F_{1} V(0)} \leq \frac{1}{W(0)},
$$

since $\sup _{x \in \mathbb{R}_{+}} x \cot ^{-1}(x) \leq 1$.

Applying results of Lemmas 5 and 6 , we conclude the proof of Theorem 3 .

Proof of Theorem 3. Let $(V, W)$ satisfy system (2.9) corresponding to the characteristics with $\xi_{0}$. Let $(\underline{V}, \underline{W})$ be the lower solution of system (2.11) in Lemma 5 with $\underline{V}(0)=V(0)$ and $\underline{W}(0)=W(0)$. Let $\bar{V}$ be the upper solution of equation $(2.20)$ in Lemma 6 with $\bar{V}(0)=V(0)$. Let $\underline{t}_{*}$ be the blow-up time of the lower solution and $\bar{t}_{*}$ be the blow-up time of the upper solution.

The upper bound for the solution of system (2.9) follows from the comparison principle for the differential equations since

$$
|V W+U|=\left(V^{2}+1\right)|U| \leq\left(V^{2}+1\right) F_{1}
$$

which implies that $V(t) \leq \bar{V}(t)$ for all $t \in\left[0, \bar{t}_{*}\right)$, for which $\bar{V}(t)$ remains bounded.

To obtain the lower bound, we note that

$$
V \geq \underline{V}, W \geq \underline{W} \Rightarrow\left\{\begin{array}{l}
\dot{V} \geq V W-F_{1} \geq \underline{V W}-F_{1}=\underline{\dot{V}}, \\
\dot{W}+F_{0} V \geq W^{2} \geq \underline{W^{2}}=\underline{\dot{W}}+F_{0} \underline{V} .
\end{array}\right.
$$

Let $\mathbf{V}=[V, W]^{T}, \underline{\mathbf{V}}=[\underline{V}, \underline{W}]^{T}$, and

$$
L=\left[\begin{array}{cc}
0 & 0 \\
F_{0} & 0
\end{array}\right]
$$

be a nilpotent matrix of order one, so that $e^{t L}=I+t L$. Thus, we write

$$
V \geq \underline{V}, W \geq \underline{W} \Rightarrow \frac{d}{d t}\left(e^{t L} \mathbf{V}\right) \geq \frac{d}{d t}\left(e^{t L} \underline{\mathbf{V}}\right)
$$

Integrating this equation in $t>0$, we infer that

$$
e^{t L} \mathbf{V}(t) \geq e^{t L} \underline{\mathbf{V}}(t)
$$

and since $e^{t L}$ is invertible for any $t \in \mathbb{R}$, we conclude that

$$
V(t) \geq \underline{V}(t), \quad W(t) \geq \underline{W}(t),
$$

for all $t \in[0, T) \subset\left[0, \underline{t}_{*}\right)$, for which $(V, W)$ remain finite. Therefore, $(V, W)$ become infinite as $t \uparrow T$ and $T \in\left[\bar{t}_{*}, \underline{t}_{*}\right]$. 
REMARK 3. The bounds on $\underline{t}_{*}$ and $\bar{t}_{*}$ in Lemmas 5 and 6 are inconclusive to compare $T$ with the exact time of blow-up $T_{0}:=\frac{1}{W(0)}$ along a particular characteristic of the dispersionless advection equation (1.2).

\section{Wave breaking in a periodic domain}

Consider now the Cauchy problem for the short-pulse equation (1.1) in a periodic domain

$$
\begin{cases}u_{t}=\frac{1}{2} u^{2} u_{x}+\partial_{x}^{-1} u, & x \in \mathbb{S}, t>0, \\ u(x, 0)=u_{0}(x), & x \in \mathbb{S},\end{cases}
$$

where $\mathbb{S}$ is a unit circle equipped with periodic boundary conditions and $\partial_{x}^{-1}$ is the mean-zero anti-derivative in the form

$$
\partial_{x}^{-1} u:=\int_{0}^{x} u\left(x^{\prime}, t\right) d x^{\prime}-\int_{\mathbb{S}} \int_{0}^{x} u\left(x^{\prime}, t\right) d x^{\prime} d x
$$

Local well-posedness and useful conserved quantities for the Cauchy problem (3.1) in a periodic domain are obtained in the following lemma.

LEMMA 7. Assume that $u_{0} \in H^{s}(\mathbb{S}), s \geq 2$ and $\int_{\mathbb{S}} u_{0}(x) d x=0$. Then there exist a maximal time $T>0$ such that the Cauchy problem (3.1) admits a unique solution

$$
u(t) \in C\left([0, T), H^{s}(\mathbb{S})\right) \cap C^{1}\left([0, T), H^{s-1}(\mathbb{S})\right)
$$

satisfying $u(x, 0)=u_{0}(x)$ and $\int_{\mathbb{S}} u(x, t) d x=0$ for all $t \in[0, T)$. Moreover, the solution $u(t)$ depends continuously on the initial data $u_{0}$ and the quantities

$$
E_{0}=\int_{\mathbb{S}} u^{2} d x, \quad E_{1}=\int_{\mathbb{S}} \sqrt{1+u_{x}^{2}} d x
$$

are constant for all $t \in[0, T)$.

Proof. Existence of the solution $u(x, t)$ and continuous dependence on $u_{0}$ is proved on $\mathbb{S}$ similarly to what is done in Lemma 1 on $\mathbb{R}$. To prove the zero-mass constraint, we note

$$
u_{t}(t) \in C\left((0, T), H^{s-1}(\mathbb{S})\right), \quad u^{2} u_{x}(t) \in C\left([0, T), H^{s-1}(\mathbb{S})\right),
$$

so that for all $t \in(0, T)$, we have

$$
\int_{\mathbb{S}} u(x, t) d x=\int_{\mathbb{S}} u_{t x} d x+\frac{1}{2} \int_{\mathbb{S}}\left(u^{2} u_{x}\right)_{x} d x=0 .
$$

Initial values of $E_{0}$ and $E_{1}$ are bounded if $u_{0} \in H^{s}(\mathbb{S}), s \geq 2$. Conservation of $E_{0}$ and $E_{1}$ on $[0, T)$ follows from the balance equations

$$
\begin{aligned}
\left(u^{2}\right)_{t} & =\left(\left(\partial_{x}^{-1} u\right)^{2}+\frac{1}{4} u^{4}\right)_{x}, \\
\left(\sqrt{1+u_{x}^{2}}\right)_{t} & =\left(\frac{1}{2} u^{2} \sqrt{1+u_{x}^{2}}\right)_{x},
\end{aligned}
$$

thanks to the continuity and the periodic boundary conditions for $\partial_{x}^{-1} u(t) \in$ $C\left((0, T), H^{s+1}(\mathbb{S})\right), u(t) \in C\left((0, T), H^{s}(\mathbb{S})\right)$, and $u_{x}(t) \in C\left((0, T), H^{s-1}(\mathbb{S})\right)$ in $x$ on $\mathbb{S}$ if $s \geq 2$. 
REMARK 4. The assumption $\int_{\mathbb{S}} u_{0}(x) d x=0$ on the initial data $u_{0}$ in the periodic domain $\mathbb{S}$ is necessary as it follows from the following apriori estimate

$$
\left|\int_{\mathbb{S}} u(x, t) d x-\int_{\mathbb{S}} u_{0}(x) d x\right| \leq\left\|u(t)-u_{0}\right\|_{L^{2}(\mathbb{S})}, \quad \forall t \in(0, T) .
$$

Note that $\int_{\mathbb{S}} u(x, t) d x=0$, for all $t \in(0, T)$ and $u(t) \in C\left([0, T), H^{s}(\mathbb{S})\right), s \geq 2$. Hence the above estimate implies that $\int_{\mathbb{S}} u_{0}(x) d x=0$. Note that no zero-mass constraint is necessary on an infinite line in Theorem 1.

The blow-up scenario for the solutions to the Cauchy problem (3.1) coincides with the one in Lemma 2 after the change of $\mathbb{R}$ by $\mathbb{S}$. The main result of this section is the proof of the finite-time wave breaking in a periodic domain, according to the following theorem.

THEOREM 4. Let $u_{0} \in H^{2}(\mathbb{S})$ and $\int_{\mathbb{S}} u_{0}(x) d x=0$. Assume that there exists $x_{0} \in \mathbb{R}$ such that $u_{0}\left(x_{0}\right) u_{0}^{\prime}\left(x_{0}\right)>0$ and

$$
\begin{array}{rlrl}
\text { either } & & \left|u_{0}^{\prime}\left(x_{0}\right)\right|>\left(\frac{E_{1}^{2}}{4 E_{0}^{1 / 2}}\right)^{1 / 3}, \\
& \left|u_{0}\left(x_{0}\right)\right|\left|u_{0}^{\prime}\left(x_{0}\right)\right|^{2}>E_{1}+\left(2 E_{0}^{1 / 2}\left|u_{0}^{\prime}\left(x_{0}\right)\right|^{3}-\frac{1}{2} E_{1}^{2}\right)^{1 / 2}, \\
\text { or } & \left|u_{0}^{\prime}\left(x_{0}\right)\right| \leq\left(\frac{E_{1}^{2}}{4 E_{0}^{1 / 2}}\right)^{1 / 3}, \quad\left|u_{0}\left(x_{0}\right)\right|\left|u_{0}^{\prime}\left(x_{0}\right)\right|^{2}>E_{1} .
\end{array}
$$

Then there exists a finite time $T \in(0, \infty)$ such that the solution $u(t) \in C\left([0, T), H^{2}(\mathbb{S})\right)$ of the Cauchy problem (3.1) blows up with the property

$$
\lim _{t \uparrow T} \sup _{x \in \mathbb{S}} u(x, t) u_{x}(x, t)=+\infty, \quad \text { while } \quad \lim _{t \uparrow T}\|u(\cdot, t)\|_{L^{\infty}} \leq E_{1} .
$$

Proof. Let $T>0$ be the maximal time of existence of the solution $u(t) \in$ $C\left([0, T), H^{2}(\mathbb{S})\right)$ to the Cauchy problem (3.1) constructed in Lemma 7 . Since $\int_{\mathbb{S}} u(x, t) d x=0$, for each $t \in[0, T)$ there is a $\xi_{t} \in[0,1]$ such that $u\left(\xi_{t}, t\right)=0$. Then for $x \in \mathbb{S}$ and $t \in[0, T)$, we have

$$
|u(x, t)|=\left|\int_{\xi_{t}}^{x} u_{x}(x, t) d x\right| \leq \int_{\mathbb{S}}\left|u_{x}(x, t)\right| d x \leq E_{1} .
$$

Since $\partial_{x}^{-1} u(t) \in C\left([0, T), H^{3}(\mathbb{S})\right)$ is the mean-zero periodic function of $x$ for each $t \in[0, T)$, there exists another $\tilde{\xi}_{t} \in[0,1]$ such that $\partial_{x}^{-1} u\left(\tilde{\xi}_{t}, t\right)=0$. Then for $x \in \mathbb{S}$ and $t \in[0, T)$, we have

$$
\left|\partial_{x}^{-1} u(x, t)\right|=\left|\int_{\tilde{\xi}_{t}}^{x} u(x, t) d x\right| \leq \int_{\mathbb{S}}|u(x, t)| d x \leq \sqrt{E_{0}} .
$$

Therefore, bounds (2.4) are rewritten with

$$
F_{0}:=\sqrt{E_{0}}, \quad F_{1}:=E_{1}
$$

The rest of the proof follows the proof of Theorem 3 . 


\section{Numerical evidence of wave breaking}

The goal of this section is to complement the analytic results by several examples and numerical computations. More specifically, we first show that the sufficient condition for wave breaking in Theorem 3 is not satisfied for the exact modulated pulse solution to the short-pulse equation which is known to remain globally bounded in space and time. Then we consider the interplay between global wellposedness and wave breaking of Theorems 2 and 3 for a class of decaying data on an infinite line. Finally, we perform numerical simulations in a periodic domain for a simple harmonic initial data and thus give illustrations to the sufficient condition for wave breaking in Theorem 4 .

Theorem 3 gives a sufficient condition for formation of shocks in the short-pulse equation (1.1) on the infinite line. Let us show that this condition is not satisfied for exact modulated pulse solutions obtained in $[\mathbf{8}, \mathbf{1 0}]$. The simplest one-pulse solution is given in the parametric form

$$
u(x, t)=U(y, t), \quad x=X(y, t),
$$

where

$$
\left\{\begin{array}{l}
U(y, t)=4 m n \frac{m \sin \psi \sinh \phi+n \cos \psi \cosh \phi}{m^{2} \sin ^{2} \psi+n^{2} \cosh ^{2} \phi}, \\
X(y, t)=y+2 m n \frac{m \sin 2 \psi-n \sinh 2 \phi}{m^{2} \sin ^{2} \psi+n^{2} \cosh ^{2} \phi},
\end{array} \quad(y, t) \in \mathbb{R}^{2},\right.
$$

$m \in(0,1)$ is an arbitrary parameter, $n=\sqrt{1-m^{2}}$, and

$$
\phi=m(y+t), \quad \psi=n(y-t) .
$$

The pulse solution enjoys the periodicity property

$$
\left\{\begin{array}{l}
U(y, t)=U\left(y-\frac{\pi}{m}, t+\frac{\pi}{m}\right), \\
X(y, t)=X\left(y-\frac{\pi}{m}, t+\frac{\pi}{m}\right)+\frac{\pi}{m}
\end{array} \quad(y, t) \in \mathbb{R}^{2} .\right.
$$

and an exponential decay in any direction transverse to the anti-diagonal on the $(y, t)$-plane.

Since

$$
\frac{\partial X}{\partial y}=1-\frac{8 m^{2} n^{2} \sin ^{2} \psi \cosh ^{2} \phi}{\left(m^{2} \sin ^{2} \psi+n^{2} \cosh ^{2} \phi\right)^{2}}=\cos \left(4 \arctan \frac{m \sin \psi}{n \cosh \phi}\right),
$$

the function $x=X(y, t)$ is invertible in $y$ for all $t \in \mathbb{R}$ if

$$
\left|\frac{m \sin \psi}{n \cosh \phi}\right|<\tan \frac{\pi}{8} \Rightarrow \frac{m}{n} \leq \tan \frac{\pi}{8},
$$

that is for all $m \in\left(0, m_{c r}\right)$, where $m_{c r}=\sin \frac{\pi}{8} \approx 0.383$. For these values of $m$, the pulse solution $u(x, t)$ is analytic in variables $(x, t) \in \mathbb{R}$, has the space-time periodicity

$$
u(x, t)=u\left(x-\frac{\pi}{m}, t+\frac{\pi}{m}\right), \quad(x, t) \in \mathbb{R},
$$

and the exponential decay in the transverse direction to the anti-diagonal in the $(x, t)$-plane. The graph of a nonsingular pulse solution for $m=0.32$ is shown on Figure 2 (left). 
REMARK 5. Coordinate $y$ in the exact solution (4.1) is different from coordinate $\xi$ in the method of characteristics because $X(y, 0) \neq y$. Nevertheless, $X(y, t)$ and $U(y, t)$ satisfy the same set of equations

$$
\frac{\partial X}{\partial t}=-\frac{1}{2} U^{2}(y, t), \quad \frac{\partial U}{\partial t}=\left.\partial_{x}^{-1} u\right|_{x=X(y, t)}
$$

so that $\xi$ and $y$ are uniquely related by the representation $\xi=X(y, 0)$. If $y$ is found as a function of $\xi$, the initial data of the Cauchy problem (2.1) is found from $u_{0}(\xi)=U(y, 0)$.

Since $u(x, t)$ is analytic in $x \in \mathbb{R}$ for any fixed $t \in \mathbb{R}$ and decays to zero exponentially fast at infinity, it is clear that $u(\cdot, t) \in H^{2}(\mathbb{R})$. Furthermore, since $\partial_{x}^{-1} u=u_{t}-\frac{1}{2} u^{2} u_{x}$, it also follows that $u(\cdot, t) \in \dot{H}^{-1}(\mathbb{R})$. We compute numerically bounds $F_{0}$ and $F_{1}$ using the exact solution (4.1) and new definitions

$$
F_{1}:=\sup _{t \in \mathbb{R}}\|u(\cdot, t)\|_{L^{\infty}}, \quad F_{0}:=\sup _{t \in \mathbb{R}}\left\|\partial_{x}^{-1} u(\cdot, t)\right\|_{L^{\infty}} .
$$

It follows from Remark 1 that bounds $F_{1}$ and $F_{0}$ defined above preserve the sufficient condition of Theorem 3 with respect to the scaling transformation (1.8).

Let us define

$$
\begin{aligned}
& f_{1}:=\sup _{x \in I_{1}}\left[\left|u_{0}(x)\right|\left|u_{0}^{\prime}(x)\right|^{2}-F_{1}\right], \\
& f_{2}:=\sup _{x \in I_{2}}\left[\left|u_{0}(x)\right|\left|u_{0}^{\prime}(x)\right|^{2}-F_{1}-\left(2 F_{0}\left|u_{0}^{\prime}(x)\right|^{3}-\frac{1}{2} F_{1}^{2}\right)^{1 / 2}\right],
\end{aligned}
$$

where

$$
\begin{aligned}
& I_{1}=\left\{x \in \mathbb{R}:\left|u_{0}^{\prime}(x)\right| \leq\left(\frac{F_{1}^{2}}{4 F_{0}}\right)^{1 / 3}, u_{0}(x) u_{0}^{\prime}(x)>0,\right\}, \\
& I_{2}=\left\{x \in \mathbb{R}:\left|u_{0}^{\prime}(x)\right|>\left(\frac{F_{1}^{2}}{4 F_{0}}\right)^{1 / 3}, u_{0}(x) u_{0}^{\prime}(x)>0,\right\} .
\end{aligned}
$$

According to Theorem 3, wave breaking occurs if either $f_{1}$ or $f_{2}$ is positive. For the exact modulated pulse solution (4.1) at $t=0$, the numerical calculations show that the set $I_{2}$ is empty and the quantity $f_{1}$ is strictly negative for any $m \in\left(0, m_{c r}\right)$, see Figure 2 (right). Therefore, the sufficient condition for the wave breaking in Theorem 3 is not satisfied, which corresponds to our understanding that the exact modulated pulse solutions (4.1) remain bounded for all $(x, t) \in \mathbb{R}^{2}$. We note, however, that the sufficient condition for the global well-posedness in Theorem 2 is satisfied only for pulses with $m \in\left(0, \frac{1}{32}\right)$, since $2 \sqrt{2 E_{1} E_{2}}=32 m$. This computation shows that the sufficient condition of Theorem 2 is not sharp.

Next we compare the sufficient conditions for the global well-posedness and wave breaking in Theorems 2 and 3 for a class of initial data

$$
u_{0}(x)=a\left(1-2 b x^{2}\right) e^{-b x^{2}}, \quad a>0, \quad b>0,
$$

where parameters $a$ and $b$ determine the amplitude and steepness of $u_{0}$. Note that the zero-mass constraint $(2.2)$ is satisfied by $u_{0}$ and it is clear that $u_{0} \in H^{2}(\mathbb{R}) \cap$ $\dot{H}^{-1}(\mathbb{R})$. The conserved quantities $E_{-1}$ and $E_{0}$ can be computed analytically,

$$
E_{-1}=\frac{a^{2} \sqrt{\pi}\left(256 \sqrt{2}-51 a^{2} b\right)}{2048 \sqrt{b^{3}}}, \quad E_{0}=\frac{3 a^{2} \sqrt{2 \pi}}{8 \sqrt{b}},
$$



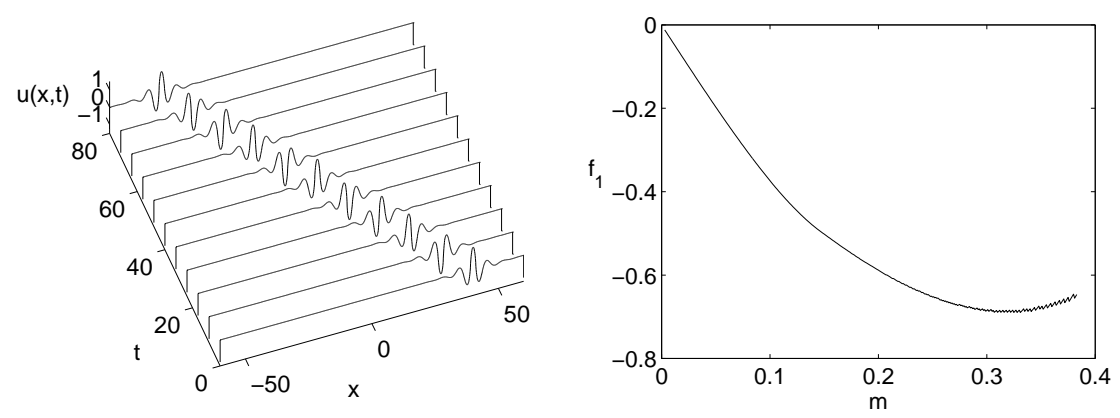

Figure 2. The exact modulated pulse solution (4.1) of the shortpulse equation (1.1) for $m=0.32$ (left). The quantity $f_{1}$ is negative for any $m \in\left(0, m_{c r}\right)$ (right).

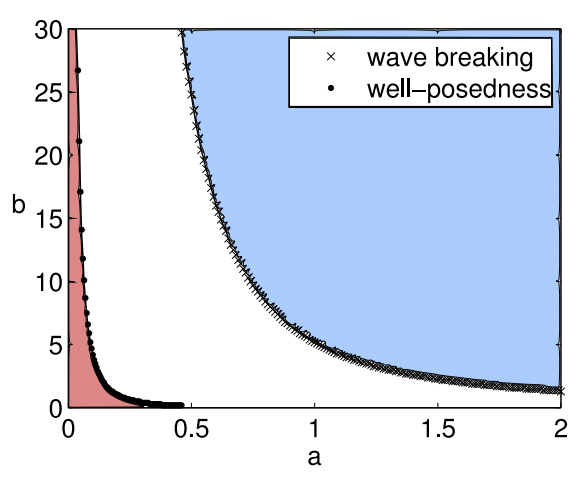

FiguRE 3. Boundaries of the global well-posedness and the wave breaking in the Cauchy problem (2.1) with initial data (4.3): the global well-posedness occurs below the lower curve and the wave breaking occurs above the upper curve.

whereas the conserved quantities $E_{1}$ and $E_{2}$ are not expressed in a closed form. Using numerical approximations of the integrals, we determine the boundary of the well-posedness region in the $(a, b)$-plane by finding the parameters $a$ and $b$ from the condition $2 \sqrt{2 E_{1} E_{2}}=1$. We also compute the boundary of the wave breaking region in the $(a, b)$-plane by computing $f_{1}$ and $f_{2}$ in (4.2). Unlike the case of modulated pulses, we find that the set $I_{1}$ is empty and $f_{2}$ may change the sign along the curve on the $(a, b)$-plane. The two boundaries are shown on Figure 3, where we can see that the two regions of global well-posedness and wave breaking are disjoint.

Finally, we perform numerical simulations of the periodic Cauchy problem (3.1) with the 1-periodic initial data

$$
u_{0}(x)=a \cos 2 \pi x, \quad a>0 .
$$



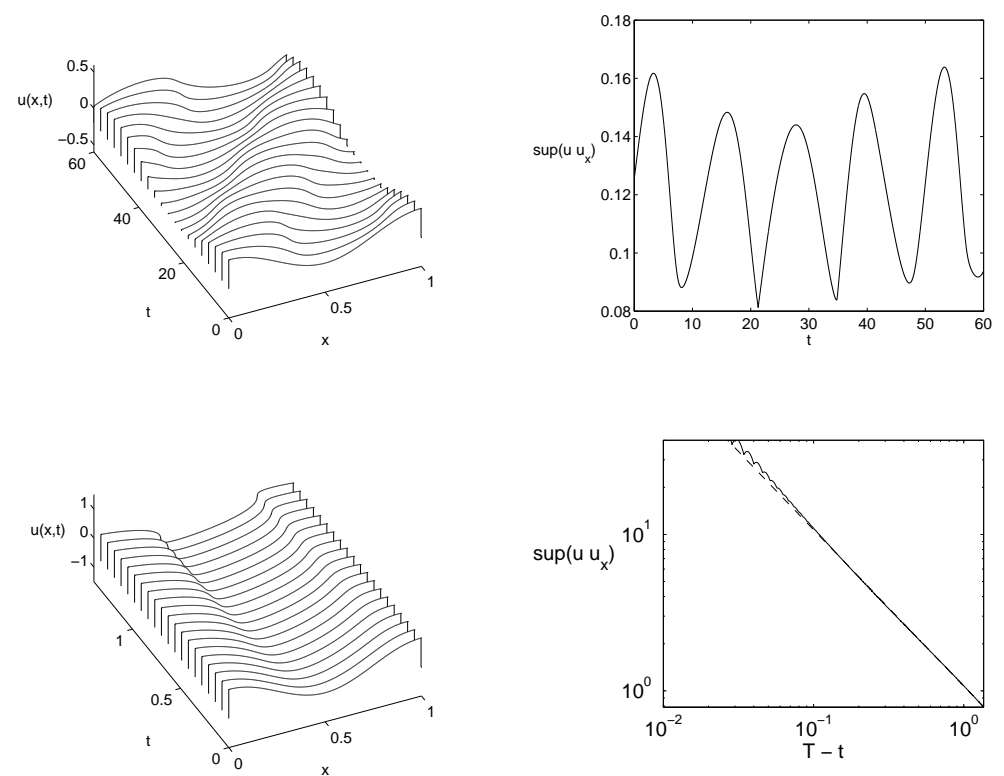

FiguRE 4. Solution surface $u(x, t)$ (left) and the supremum norm $W(t)$ (right) for $a=0.2$ (top) and $a=0.5$ (bottom). The dashed curve on the bottom right picture shows the linear regression with $C=1.072, T=1.356$.
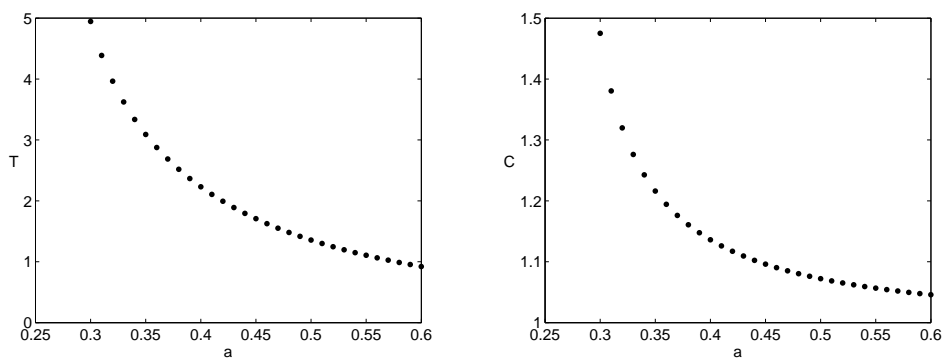

Figure 5. Time of wave breaking $T$ versus $a$ (left). Constant $C$ of the linear regression versus $a$ (right).

The two conserved quantities $E_{0}$ and $E_{1}$ in Lemma 7 are computed analytically as

$$
E_{0}=\frac{1}{2} a^{2}, \quad E_{1}=\frac{2}{\pi} \mathbf{E}(2 \pi a i),
$$

where $\mathbf{E}$ stands for a complete elliptic integral. Using the above conserved quantities we find out that the sufficient condition for the wave breaking in Theorem 4 is satisfied for $a>1.053$. 
To illustrate the behaviour of a solution $u(x, t)$ to the Cauchy problem (3.1), we perform numerical simulations using a pseudospectral method. When the parameter $a$ is sufficiently small, the value of

$$
W(t):=\sup _{x \in \mathbb{S}} u(x, t) u_{x}(x, t)
$$

remains bounded as shown on the top panel of Figure 4 for $a=0.2$. On the other hand, when $a$ becomes larger, the wave breaking occurs, as on the bottom panel of Figure 4 for $a=0.5$. On the bottom right panel of Figure 4 we show using the linear regression that the curve $W^{-1}(t)$ is fitted well with the straight line $A+B t$ for some coefficients $(A, B)$. Thus, we make a conclusion that

$$
W(t) \simeq \frac{C}{T-t} \quad \text { for } \quad 0<T-t \ll 1
$$

where $C=-B^{-1}$ and $T=-A B^{-1}$. Using the linear regression, we also obtain pairs $(T, C)$ for different values of $a$. The results are shown on Figure 5 . Note that the constant $C$ approach 1 as $a$ gets larger. This observation is consistent with the exact blow-up law $W(t)=\frac{1}{T-t}$ obtained for the advection equation (1.2) using the method of characteristics.

\section{References}

[1] Y. Chung, C.K.R.T. Jones, T. Schäfer, And C.E. Wayne, Ultra-short pulses in linear and nonlinear media, Nonlinearity 18, 1351-1374 (2005)

[2] A. Constantin, J. Escher, Well-posedness, global existence, and blowup phenomenon for a periodic quasi-linear hyperbolic equation, Comm. Pure Appl. Math., 51 (1998), 475-504.

[3] A. Constantin and J. Escher, Wave breaking for nonlinear nonlocal shallow water equations, Acta Mathematica, 181, 229-243 (1998).

[4] D. Chae, A. Cordoba, D. Cordoba, and M.A. Fontelos, Finite time singularities in a 1D model of the quasi-geostrophic equation, Adv. Math., 194, 203-223 (2005).

[5] J. HunteR, Numerical solutions of some nonlinear dispersive wave equations, Lectures in Appl. Math., 26 (1990), 301-316.

[6] Y. LiU And Z.Y. Yin, Global existence and blow-up phenomena for the DegasperisProcesi equation, Comm. Math. Phys. 267, 801-820 (2006).

[7] Y. LiU And Z.Y. Yin, On the blow-up phenomena for the Degasperis-Procesi equation, Inter. Math. Res. Not. 117, 22 pages (2007).

[8] Y. Matsuno, Periodic solutions of the short pulse model equation, J. Math. Phys. 49, 073508, 18 pp. (2008)

[9] D. Pelinovsky, A. Sakovich, Global well-posedness of the short-pulse and sineGordon equations in energy space, arXiv: 0809.5052 (2008), 14 pages.

[10] A. SAKovich And S. SAKOvich, Solitary wave solutions of the short pulse equation, J. Phys. A: Math. Gen. 39, L361-L367 (2006).

[11] T. Schäfer AND C. E. Wayne, Propagation of ultra-short optical pulses in cubic nonlinear media, Physica D, 196 (2004), 90-105.

[12] Z. YIN, On the Cauchy problem for an integrable equation with peakon solutions, Illinois J. Math. 47, 649-666 (2003). 
Department of Mathematics, University of Texas at Arlington, Arlington, TX, 76019, USA

E-mail address: yliu@uta.edu

Department of Mathematics, McMaster University, Hamilton, ON, L8S 4K1, Canada

E-mail address: dmpeli@math.mcmaster.ca

Department of Mathematics, McMaster University, Hamilton, ON, L8S 4K1, Canada

E-mail address: sakovias@math.mcmaster.ca 